\title{
Seroprevalence of Hepatitis E Among Hemodialysis Patients: A Report From Hamadan, Iran
}

\author{
Peyman Eini ${ }^{1}$; Mojgan Mamani ${ }^{1, *} ;$ Marzieh Javani $^{2}$

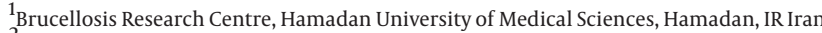 \\ ${ }^{2}$ Department of Psychology, Qazvin University of Medical Sciences, Qazvin, IR Iran \\ *Corresponding Author: Mojgan Mamani, Brucellosis Research Centre, Hamadan University of Medical Sciences, P. O. Box: 65168, Hamadan, IR Iran. Tel: +98-8138274192, Fax: \\ +98-8138276010, E-mail: mojganmamani@gmail.com
}

Received: December 21, 2014; Revised: March 10, 2015; Accepted: April 11, 2015

Background: Previous studies have documented a high prevalence of hepatitis E among patients undergoing maintenance hemodialysis. Available studies reporting on the seroprevalence of hepatitis $\mathrm{E}$ in hemodialysis patients in Iran, an endemic region for the disease, are sparse.

Objectives: The present study aimed to determine the prevalence rate of anti-hepatitis E antibody in hemodialysis patients in Hamadan, Iran.

Patients and Methods: In this cross-sectional study, all 153 consecutive patients undergoing hemodialysis in two centers were enrolled. Patients' demographic and clinical data were collected, using a standard questionnaire and from medical records. Serum immunoglobulin $G$ concentrations against hepatitis $E$ were determined using the enzyme linked immunosorbent assay method.

Results: Thirty patients (19.2\%), were seropositive. Seropositive patients were not significantly different from seronegative patients, with regard to age, sex, level of education, access to filtered water, and duration and frequency of hemodialysis. The proportions of patients with hepatitis B, C, and HIV infection were comparable between the two groups.

Conclusions: One in five patients undergoing maintenance dialysis in Hamadan is seropositive for hepatitis E immunoglobulin G antibody. Future studies are needed to investigate the factors contributing to the observed high prevalence rate and the possibility of parenteral transmission of hepatitis $\mathrm{E}$.

Keywords: Hepatitis E; Seroprevalence; Immunoglobulin; Renal Dialysis

\section{Background}

Hepatitis E Virus (HEV), the sole member of the family Hepeviridae, is an under-recognized cause of acute hepatitis. In resource-poor areas of developing countries, where the virus is endemic, HEV is responsible for large water-borne outbreaks of significant morbidity. In nonendemic areas, where sanitary standards are optimal, HEV has been implicated in sporadic cases of hepatitis (1). Studies over the past three decades, have substantially transformed our understanding of the pathogen and its true burden. The HEV is now believed to be the most common etiologic agent in acute hepatitis around the world (2).

The HEV induced hepatitis is usually a self-limiting disease, with manifestations that are often subclinical. It is not distinguishable from other causes of acute hepatitis, and most of the times, the symptoms tend to resolve spontaneously. The disease associated with low morbidity and mortality (1). Since the clinical picture and alterations in laboratory parameters are not specific in the hepatitis caused by HEV, diagnosis should be confirmed by documenting elevated titers of immunoglobulin $\mathrm{M}$ $(\operatorname{IgM})$ in the serum, the rise of which coincide with the onset of clinical symptoms (2). About 6 weeks after the exposure, IgM titers start to fall, whereas Immunoglobulin $\mathrm{G}$ (IgG) levels continually increase and remain detectable long after the recovery from the acute infection (2).

Immunocompromised patients are at an increased risk for severe infection, protracted viremia, and disease chronicity, which can ultimately lead to cirrhosis and endstage liver failure (3). In patients with end-stage renal disease (ESRD), several immune functions, especially those relating to the innate, as well as the adaptive immunity, are markedly impaired (4). Consequently, patients are more susceptible to acquisition of infections, including viral hepatitis.

\section{Objectives}

Fecal-oral transmission (contamination of water sources with fecal material) has been described as the main

Copyright ( ) 2015, Kowsar Corp. This is an open-access article distributed under the terms of the Creative Commons Attribution-NonCommercial 4.0 International License (http://creativecommons.org/licenses/by-nc/4.0/) which permits copy and redistribute the material just in noncommercial usages, provided the original work is properly cited. 
route of HEV transmission. In patients undergoing hemodialysis (HD), the possibility of a person-to-person nosocomial transmission via infected blood or contamination of devices/intravenous sets has been raised, further highlighting that the epidemiology and clinical features of HEV among HD patients may be different from the general population.

Despite these considerations, studies investigating the epidemiology of HEV seropositivity among HD patients are sparse and the findings are often conflicting. To address the knowledge gap in the seroprevalence of HEV among HD patients in Iran, an endemic country for HEV, the present study was designed and conducted. Here, we delineate the prevalence rate of anti-HEV antibody in HD patients and compare our findings with the rates observed in the general population and also, HD units across the country.

\section{Patients and Methods}

\subsection{Patients}

Between March and September 2010, all patients with ESRD undergoing maintenance HD in two dialysis units in the city (Be'sat Hospital and Shahid Beheshti Hospital, Hamadan, Iran), were consecutively recruited.

Recruited patients were interviewed and data regarding age, sex, education, duration and frequency of dialysis were recorded in pre-designed questionnaires. Data regarding previous infection with hepatitis $B$, hepatitis $C$, and human immunodeficiency virus (HIV) were retrieved from patients' hospital records and were recorded in the questionnaires.

All procedures dealing with human subjects were conducted in accordance with the guidelines and standards laid down in the declaration of Helsinki. The ethics committee of the Hamadan University of Medical Sciences read and approved the study protocol. Written informed consent was obtained from each patient, prior to enrollment.

\subsection{Laboratory Assessment}

Immediately before a HD session, a sample of $3 \mathrm{~mL}$ of venous blood was obtained from each participant. Specimens were then sent to hospital laboratory, where they were centrifuged and stored in $18^{\circ} \mathrm{C}$, until assessment. Serum IgG concentrations against HEV were determined using the enzyme linked immunosorbent assay (ELISA) method, with commercial kits (Diagnostic BioProbes s.r.l., Milan, Italy). The distinction between positive and negative cases was done according to the manufacturer's instructions and using the provided cut-off in the product brochure.

\subsection{Statistical Analysis}

All statistical analyses were performed using the Statistical Package for Social Sciences (SPSS®) version 17
(SPSS Inc., Chicago, Illinois). Categorical variables are presented as proportions. The prevalence rate for HEV seropositivity was calculated by direct counting and the $95 \%$ confidence interval ( $95 \% \mathrm{CI}$ ) for the prevalence were calculated by the modified Wald method, developed by Agresti and Coull (5). To investigate the distribution of variables between seropositive and seronegative groups, contingency tables were drawn and the statistical significance of the difference was assessed using Chi square of Fisher's exact tests, where appropriate. In all tests, a $\mathrm{P}<0.05$ was set as the threshold to retain or reject the null hypothesis.

\section{Results}

The baseline characteristics of 153 patients included in the present study are presented in Table 1. Eighty-three patients were male (54.2\%) and female-to-male ratio was 1:1.2. Patients were on dialysis for an average of 33 months and received filtration for an average of nine hours per week. The majority of participants (73.9\%) had access to tap water for drinking and other purposes. Overall, 30 patients had positive results for HEV IgG antibody, giving rise to a prevalence rate of $19.2 \%$ (95\%CI:14.0 - 26.6).

Comparisons of the distribution of independent variable between seropositive and seronegative patients are presented in Table 1. Age, sex, the highest level of education achieved, and rate of access to filtered water were comparable between the two groups. Seropositive patients were not significantly different from their seronegative counterparts, in terms of duration of HD and also, the average time spent on HD per week. With regard to major blood-borne pathogens, one patient in the HEV seropositive group was positive for hepatitis B surface antigen. Four patients (one in the seropositive group and three in the seronegative group) had been diagnosed with hepatitis $C$ infection in the past. No cases of HIV infection were identified. The proportions of patients positive for viral blood-borne pathogens were not significantly different between the two groups (Table 1).

\section{Discussion}

In the present study, the seroprevalence of HEV IgG was assessed in a sample of 153 patients on maintenance HD in Hamadan, Iran. Based on our observations, the proportion of HD patients with positive HEV IgG was $19.2 \%$, which is approximately two-fold higher than the proportion documented in the general population of Nahavand [9.3\% (95\%CI: 8.2-10.9)]. Nahavand, which is a city in Hamadan province, is located 70 miles southwest of Hamadan. Taremi et al. included a random sample of 1824 inhabitants in the Nahavand County (6). Sera of patients were collected and anti-HEV IgG titers were determined by the same commercial kit used herein. A total of 170 individuals tested positive for HEV antibody (6). In the Keramat et al. 2012 study, in Hamadan, the anti-HEV IgG titers were positive only in $1.5 \%$ of 131 healthy and non-addict persons (7). 
Eini P et al.

Table 1. Baseline Characteristics of Patients on Maintenance Hemodialysis in Hamadan, Iran

\begin{tabular}{|c|c|c|c|c|}
\hline Characteristics & No. (\%) & Seropositive $^{\mathrm{a}}$ & Seronegative $^{b}$ & $\mathbf{P}$ \\
\hline Age, $y$ & & & & 0.332 \\
\hline$<20$ & $7(4.6)$ & $3(10.0)$ & $4(3.2)$ & \\
\hline $20-40$ & $38(24.8)$ & $6(20.0)$ & $32(26.0)$ & \\
\hline $40-60$ & $44(28.8)$ & $10(33.3)$ & $33(26.8)$ & \\
\hline$>60$ & $64(41.8)$ & $11(36.7)$ & $54(44.0)$ & \\
\hline Gender & & & & 0.602 \\
\hline Female & $70(45.8)$ & $15(50.0)$ & $55(44.7)$ & \\
\hline Male & $83(54.2)$ & $15(50.0)$ & $68(55.3)$ & \\
\hline Education & & & & 0.990 \\
\hline No formal education & $70(45.8)$ & $14(46.6)$ & $56(45.6)$ & \\
\hline Elementary School & $61(39.9)$ & $12(40.0)$ & $49(39.8)$ & \\
\hline High school & $10(6.5)$ & $2(6.7)$ & $8(6.5)$ & \\
\hline College/University & $12(7.8)$ & $2(6.7)$ & $10(8.1)$ & \\
\hline Water source & & & & 0.143 \\
\hline Tap water (filtered) & $113(73.9)$ & $19(63.3)$ & $94(76.4)$ & \\
\hline Well water (not filtered) & $40(26.1)$ & $11(36.6)$ & $29(25.6)$ & \\
\hline Time on dialysis, mo. & & & & 0.599 \\
\hline$<20$ & $56(36.6)$ & $13(43.3)$ & $43(34.9)$ & \\
\hline $20-40$ & $60(39.2)$ & $10(33.3)$ & $50(40.6)$ & \\
\hline $40-60$ & $10(6.5)$ & $3(10.0)$ & $7(5.7)$ & \\
\hline$>60$ & $27(17.7)$ & $4(13.3)$ & $23(18.7)$ & \\
\hline Duration of weekly dialysis, $h$ & & & & 0.870 \\
\hline$<6$ & $25(16.3)$ & $4(16.3)$ & $21(17.1)$ & \\
\hline $6-10$ & $61(39.9)$ & $12(39.9)$ & $49(39.8)$ & \\
\hline$>10$ & $67(43.8)$ & $14(43.8)$ & $53(43.1)$ & \\
\hline HBS-Ag & & & & 1.000 \\
\hline Positive & & $0(0.0)$ & $1(0.8)$ & \\
\hline Negative & & $30(100.0)$ & $122(99.2)$ & \\
\hline HCV-Ab & & & & 1.000 \\
\hline Positive & & $1(3.3)$ & $3(2.4)$ & \\
\hline Negative & & $29(96.7)$ & $120(97.6)$ & \\
\hline
\end{tabular}

$\mathrm{a} n=30$.

$\mathrm{b} \mathrm{n}=123$.

The question is why a significantly higher proportion of individuals on HD are positive for HEV antibodies, in comparison to the general population? The HEV was once believed to be transmitted solely fecal-orally due to contamination of water sources and transmission by blood did not seem to play a role in disease spread (1). Studies in the past two decades, however, have frequently raised the possibility of intravenous passage, as a route of infection, similar to that observed with hepatitis B or C (7). Several lines of evidence, in support of a possible blood-borne transmission of HEV have been accumulated (2). Cases of acute hepatitis E following transfusion with infected blood products have been documented (8). In other cases, IgG positive patients were found to have been transfused with contaminated blood in the past (9). In one neurosurgery ward in a hospital in Pakistan, an outbreak of hepatitis $\mathrm{E}$ has been traced back to a possible use of shared contaminated intravenous sets for administering mannitol and dexamethasone (10). These findings are suggestive of a parenteral route for HEV transmission and raise the possibility of presenting HEV infection, nosocomially.

On the other hand, in a retrospective cohort of individuals undergoing HD in Japan, Mitsui et al. determined se- 
rum IgG titers to HEV at baseline and after a mean follow up of 7.6 years. Of the 416 patients followed, only four new cases of positive HEV infection were identified, indicating that acquiring HEV, while being on maintenance HD, is a rare occurrence (9). Of note, one patient was transfused with two units of blood, approximately three weeks before the emergence of viremia (9).

The results from seroepidemiological surveys at Zanjan, Jahrom and Ahvaz showed various results like 26.9\%, $7 \%$, and $10.6 \%$, respectively (11-13). Our findings are higher than the rate observed in Jahrom and lower than that of Zanjan. Collectively, the current report, along with previous observations, indicates a high degree of variability in different HD centers across the country. Whether the intervariability between different studies could be ascribed to a real discrepancy in the epidemiology of HEV among Iranian HD patients or it is merely a consequence of enrollment of samples, with distinct characteristics (e.g. age, sex), or alternatively, the use of different commercial kits used, remains to be elucidated.

In the present study, no variables conferred susceptibility to or provided protection against HEV seropositivity. This is in line with the majority of previous observations, where no significant variable has emerged as a risk factor for being HEV-antibody positive (13). Moreover, we found no link between HEV seropositivity and infection with viral blood-borne infections with hepatitis B, C, or HIV. Similar findings have also been generated by other investigators $(13,14)$.

A number of limitations in the present study deserve mention. The cross-sectional nature of the study precludes us from drawing inferences of causality from the observed associations. Moreover, it is worthwhile to note that no anti-HEV assay to date has been approved by the food and drug administration and the available commercial assays vary widely, in terms of precision (15).

In spite of the aforementioned limitations, in the present study, we documented a high rate of HEV seropositivity in a sample of patients on maintenance HD, in an endemic region. The prevalence rate appears to be higher than that of the general population living in the same region.

In conclusion the prevalence of hepatitis E among HD patients in our study has been high. This can lead to the possible parenteral transmission of hepatitis $\mathrm{E}$ in $\mathrm{HD}$ patients and other high-risk individuals. We need further studies to confirm this and the obtained insights may lead to cautionary measures required for high-risk groups.

\section{Acknowledgements}

The authors thank the Research Deputyship of Hamadan University of Medical Sciences, Hamadan, Iran, for their financial support and special thanks to Mr. Abbas Moradi, for statistical analysis.

\section{Authors' Contributions}

Peyman Eini was involved in the study concept and design, the study supervision, analysis and interpretation of data. Marzieh Javani participated in study design and analyzed the data. Mojgan Mamani was involved in the study concept and design, abstracted data, wrote and prepared the manuscript. All authors provided comments and approved the final manuscript.

\section{Funding/Support}

This study was supported financially by Vice-chancellor of Research and Technology, Hamadan University of Medical Sciences, Hamadan, Iran.

\section{References}

1. Aggarwal R, Naik S. Epidemiology of hepatitis E: current status. J Gastroenterol Hepatol. 2009;24(9):1484-93.

2. Hoofnagle JH, Nelson KE, Purcell RH. Hepatitis E. N Engl J Med. 2012;367(13):1237-44.

3. Kamar N, Selves J, Mansuy JM, Ouezzani L, Peron JM, Guitard J, et al. Hepatitis E virus and chronic hepatitis in organ-transplant recipients. N Engl J Med. 2008;358(8):811-7.

4. Kato S, Chmielewski M, Honda H, Pecoits-Filho R, Matsuo S, Yuzawa Y, et al. Aspects of immune dysfunction in end-stage renal disease. Clin J Am Soc Nephrol. 2008;3(5):1526-33.

5. Agresti A, Coull BA. Approximate is Better than "Exact" for Interval Estimation of Binomial Proportions. Am Statistician. 1998;52(2):119-26.

6. Taremi M, Mohammad Alizadeh AH, Ardalan A, Ansari S, Zali MR. Seroprevalence of hepatitis E in Nahavand, Islamic Republic of Iran: a population-based study. East Mediterr Health J. 2008;14(1):157-62.

7. Keramat F, Mamani M, Samadi M, Mohammadnezhad S, Eini P, Moradi A. Seroprevalence of Hepatitis E Virus Among Injection Drug Users and Non-Injection Drug Users in Hamadan, West of Iran. Avicenna J Clin Microb Infec. 2014;1(3):1-4.

8. Boxall E, Herborn A, Kochethu G, Pratt G, Adams D, Ijaz S, et al Transfusion-transmitted hepatitis $\mathrm{E}$ in a 'nonhyperendemic country. Transfus Med. 2006;16(2):79-83.

9. Mitsui T, Tsukamoto Y, Yamazaki C, Masuko K, Tsuda F, Takahashi $\mathrm{M}$, et al. Prevalence of hepatitis E virus infection among hemodialysis patients in Japan: evidence for infection with a genotype 3 HEV by blood transfusion. J Med Virol. 2004;74(4):563-72.

10. Siddiqui AR, Jooma RA, Smego RJ. Nosocomial outbreak of hepatitis E infection in Pakistan with possible parenteral transmis sion. Clin Infect Dis. 2005;40(6):908-9.

11. Mobaien AR, Mohammadi R, Sorouri R, Sadeghi K. Hepatitis E virus seroprevalence in haemodialysis patients in Zanjan Province, Islamic Republic of Iran. East Mediterr Health J. 2013;19(7):608-12.

12. Pourahmad M, Sotoodeh AR, Nasiri H. Hepatitis E Virus Infection in Hemodialysis Patients: A Seroepidemiological Survey in Jahrom, Southern Iran. Hepat Mon. 2009;9(3):232-5.

13. Beladi Mousavi SS, Motemednia F, Beladi Mousavi M. Epidemiology of hepatitis e virus infection in patients on chronic hemodialysis. Jundishapur J Microbiol. 2014;7(5):e6993.

14. Taremi M, Khoshbaten M, Gachkar L, EhsaniArdakani M, Zali M. Hepatitis E virus infection in hemodialysis patients: a seroepidemiological survey in Iran. BMC Infect Dis. 2005;5:36.

15. Mast EE, Alter MI, Holland PV, Purcell RH. Evaluation of assays for antibody to hepatitis E virus by a serum panel. Hepatitis E Virus Antibody Serum Panel Evaluation Group. Hepatology. 1998;27(3):857-61. 